\title{
Medical Management of Cervical Ectopic Pregnancy
}

\author{
Wasima Arif ${ }^{1}$, Farrah Mukhtar $^{2}$, Iffat Naheed ${ }^{3}$, Farhat Naz ${ }^{4}$
}

\begin{abstract}
Cervical ectopic pregnancy is a rare condition with implantation in the endocervical canal below the closed internal os. It accounts for less than $1 \%$ of all ectopic pregnancies. It is associated with high morbidity and mortality. A case of cervical ectopic pregnancy is reported. She was a young nulliparous female, timely referred from peripheral hospital. She was treated successfully with medical management and thus fertility was preserved.
\end{abstract}

Keywords: Cervical ectopic pregnancy, transabdominal ultrasound, methotrexate.

Date of Submission 22-9-2015

Date of Revision Received 30-1-2016

Date of Acceptance for Publication 8-4-2016

Conflict of Interest: None

Funding Source: None

\section{Arif W.}

Senior Registrar of Obstetrics \& Gynaecology Unit - IV

Lady Aitchison Hospital, Lahore

Mukhtar F. ${ }^{2}$

PGR Obstetrics \& Gynaecology Unit - IV

Lady Aitchison Hospital, Lahore

Naheed I. ${ }^{3}$

Assistant Professor of Obstetrics \& Gynaecology Unit - IV

KEMU/ Lady Aitchison Hospital, Lahore

$\mathrm{Naz}_{\mathrm{F}}{ }^{4}$

Professor of Obstetrics \& Gynaecology Unit - IV

KEMU/ Lady Aitchison Hospital, Lahore

\section{Introduction}

Cervical ectopic pregnancy is defined as the implantation of the blastocyst in the endocervix below the internal os. ${ }^{1-4}$ It accounts for less than $1 \%$ of all ectopic pregnancies with an estimated risk of $1: 2500$ to 1 : $18000 .^{4-5}$ Cervical ectopic pregnancies are high risk cases, as they may present with an unexpected life threatening haemorrhage secondary to the erosion of cervical blood vessels, which may require hystrectomy to save the patient. Available options to control bleeding are curettage, foly catheter balloon tamponade, local prostaglandin injection, shirodkar cerclage or other cervical sutures, uterine artery or hypogastric artery ligation or more radical surgeries (hysterectomy) were the available options. ${ }^{1,2,6,7}$ Ultrasonographic diagnosis of cervical ectopic pregnancy was first described by Raskin (1978). ${ }^{8}$ Since then technical improvements in ultrasound have been crucial to an early diagnosis in nonsymptomatic women in the first trimester, thus allowing conservative management. ${ }^{1,3} \mathrm{We}$ report a case of cervical ectopic pregnancy describing the ultrasound diagnosis and successful medical management with methotrexate.

\section{Case Report}

A 25 years age woman being referred from a district hospital. She was married for six months, presented with mild lower abdominal pain and slight vaginal bleeding for 4 days. Her relevant history included gestational amenorrhoea of 8 weeks with positive urine pregnancy test. She had an ultrasound report showing suspicion of cervical ectopic pregnancy. Her previous menstrual cycle was regular. There was no history of contraceptive use. Her past medical, surgical and social histories were unremarkable. She was not anaemic. There was no tenderness in her lower abdomen. Her 
speculum examination revealed oedematous, hypraemic cervix with slight bleeding coming through external os (Figure 1).

Trans abdominal ultrasound examination revealed ballooned out cervical canal having a gestational sac with fetus of CRL $1.1 \mathrm{~cm}$ corresponding to 7 weeks and 2 days with absent fetal cardiac activity. The uterine cavity was found empty with thickened endometrium (Figure 2). A diagnosis of ectopic pregnancy was made.

Quantitative serum human chorionic gonadotrophin ( $\beta$ HCG) was $26033 \mathrm{mIU} / \mathrm{ml}$. Renal function tests, liver function tests, complete blood counts were

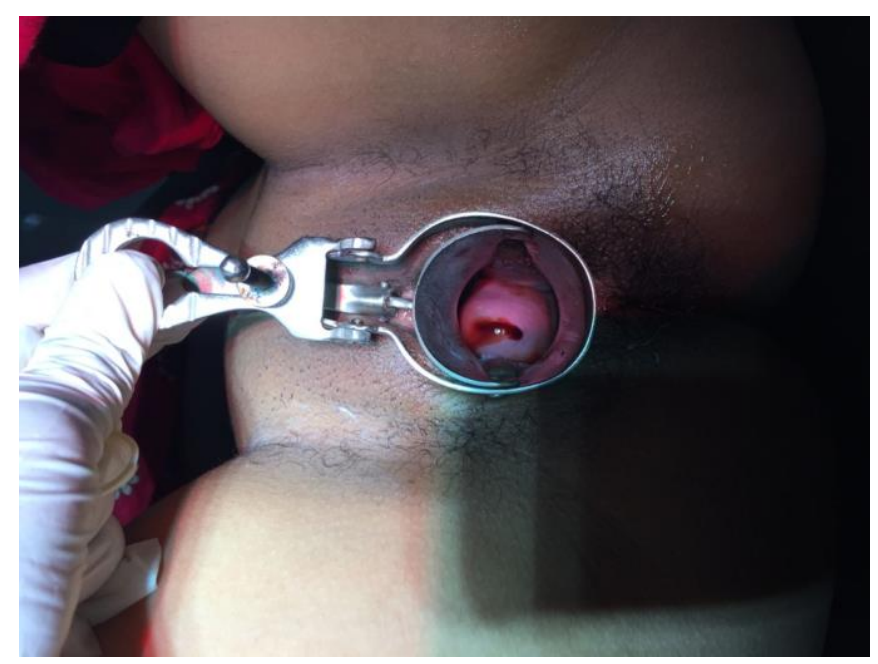

Figure 1:

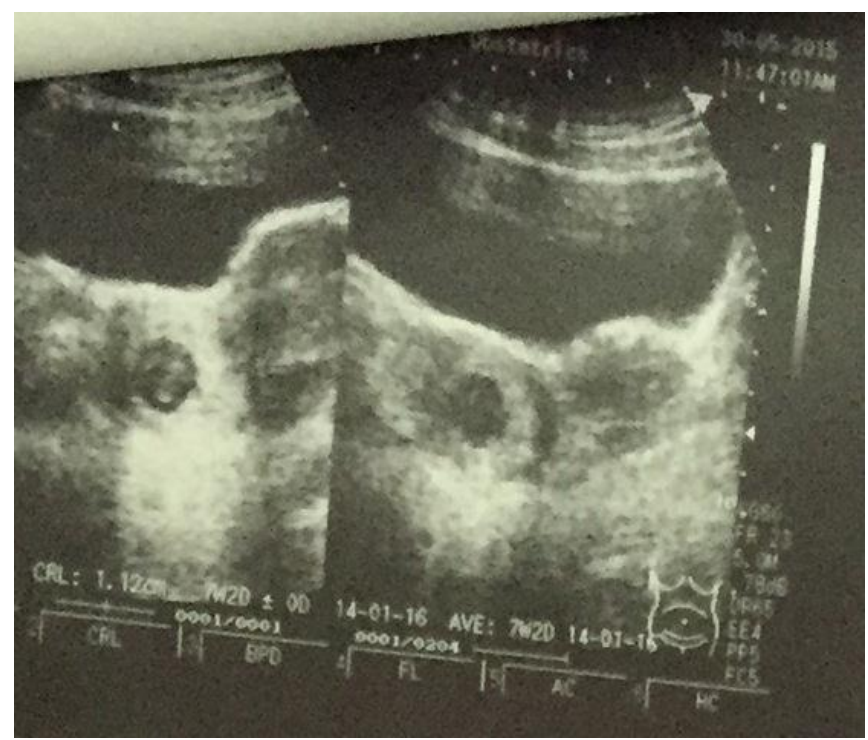

Figure 2:

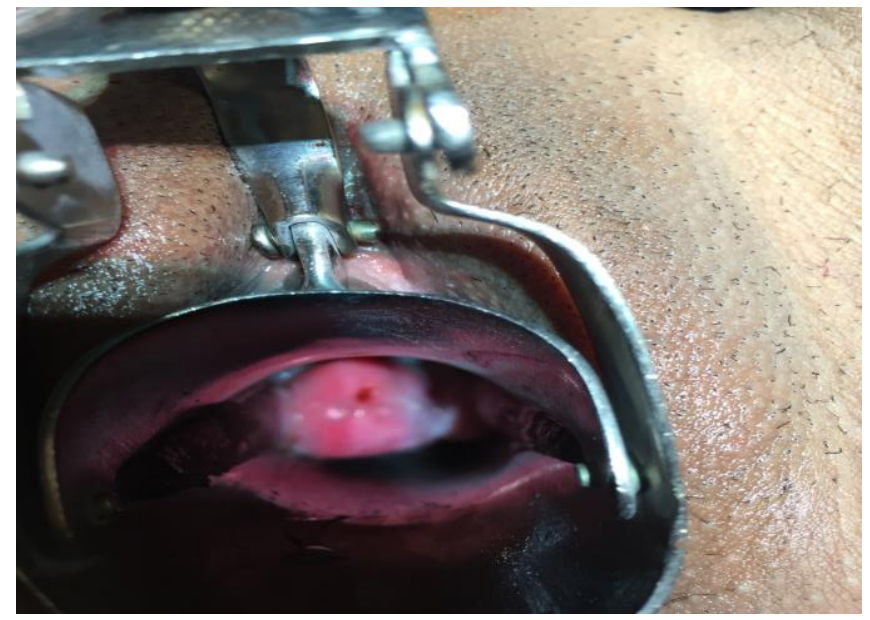

Figure 3:

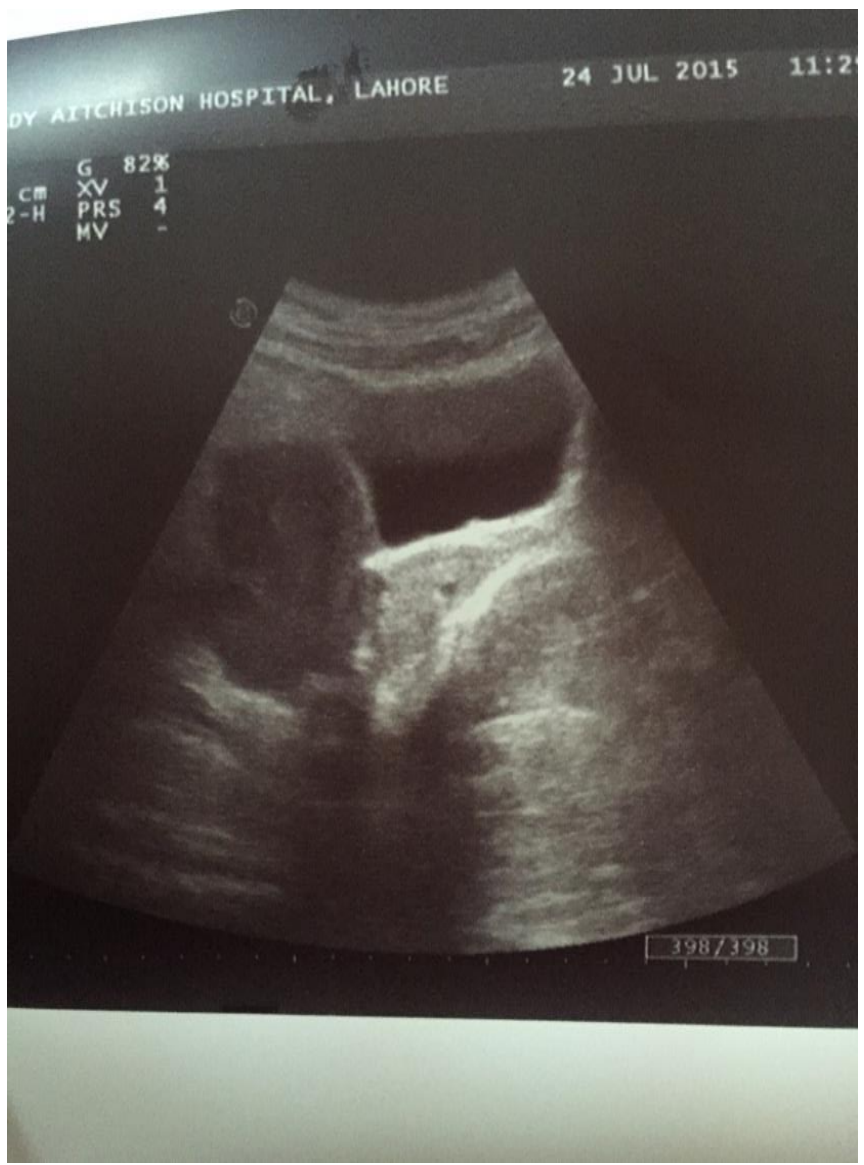

Figure 4:

done in addition to basic investigations. Screened cross matched blood was arranged.

Multi dose methotrexate regime, i.e. $1.0 \mathrm{mg} / \mathrm{kg}$ body weight was given intramuscularly on days 1,3 , 
5 , and 7 interspaced with folenic acid $0.1 \mathrm{mg} / \mathrm{kg}$ injection, i.e. $0.1 \mathrm{mg} / \mathrm{kg}$ body weight. She was weekly followed-up by symptoms of abdominal pain, vaginal bleeding, ultrasound scan and serum B HCG levels. After 8 weeks follow up she was asymptomatic. speculum examination showed normal cervix (Figure 3), review ultrasound showed absence of gestational sac in the cervical canal (Figure 4), Serum B HCG level was less than $2 \mathrm{mIU} / \mathrm{ml}$.

\section{Discussion}

The ectopic pregnancy is one of the risk factors for maternal morbidity and mortality in early pregnancy. Cervical ectopic pregnancies account for less than $1 \%$ of all ectopic pregnancies with an estimated risk of one in 2500 to one in 18,000 , $^{4,5}$

Major predisposing factors are dilatation and curettage, previous caesarean delivery and in vitro fertilezation. ${ }^{9}$ Asherman's syndrome, prior instrumentation or therapeutic abortion use, infertility, and prior ectopic pregnancies, primary embryo anomaly have also been implicated as predisposing factors. ${ }^{4}$ The rarity of the condition has prevented any retrospective studies, and the association of cervical pregnancy with all these factors remains weak. ${ }^{10,11}$ In our case, none of the above mentioned predisposing factors were found. Regarding symptoms, cervical pregnancy classically presents with painless first trimester vaginal bleeding, although several case reports describe patients with cramping pain. Our patient complained the same.

The ultrasound diagnosis of a cervical ectopic pregnancy requires visualization of an intracervical ectopic gestational sac or trophoblastic mass below a closed internal os. If internal os is not visualized, the sac should be visualized below the uterine artery insertion or vesicouterine fold. Recognizing its sonographic appearance is the first step for a correct management, because it may be mistaken for an intrauterine pregnancy, an incomplete abortion or even an endocervical cyst. $^{1,12}$ In our case, trans abdominal ultrasound approach illustrated the diagnosis, whereas trans-vaginal ultrasound seems to be the most appropriate imaging method. The specificity of three - dimensional (3D) ultrasound imaging has been reported to be better than two dimentional (2D) scans as the 3D image incorporates an additional coronal section that is not possible with 2D imaging. ${ }^{13}$ Magnetic Resonance Imaging (MRI) is helpful in doubtful cases as tissue characterization is better with MRI when compared with ultra- sound. $^{12}$

Treatment choices for cervical ectopic pregnancy may be divided into five categories ${ }^{4}$ temponade with folys catheter, reduction of blood supply, surgical excision of trophoblast, intra amniotic feticide, (potassium chloride and/or methotrexate.) The most appropriate treatment depends on the clinical presentation ${ }^{15-17}$ In a haemodynamically stable patient, medical management with single or multiple dose methotrexate (MTX) is the therapy of choice. ${ }^{18,6}$ If foetal cardiac activity is present, intra amniotic injection of potassium chloride has been associated with avoidance of hysterectomy in $80 \%$ of cases. ${ }^{19}$ Dilatation and curettage is the surgical option in such patients but it carries a significant risk of severe haemorrhage. Preoperative uterine artery embolization may be useful to help control haemorrhage. ${ }^{7}$ In haemodynamically unstable patients or those who have failed medical management, dilatation and curettage is required. The risk of haemorrhage can be reduced by trans vaginal ligation of cervical branches of uterine arteries, cervical circlage, or intracervical injection of vasopressin in addition to uterine artery embolization. In a post operative haemorrhagic patient, the treatment options are temponade with folys balloon, haemostatic suture in the implantation site, bilateral uterine or internal artery ligation, or hysterectomy. ${ }^{3}$

Methotrexate chemotherapy with either viable or nonviable cervical pregnancy at $<12$ weeks' gestation carries a high success rate (>91\%) for preservation of uterus. ${ }^{20}$ Among the various routes for methotrexate administration, intramuscular route is usually preferred. The patient should be hemodynamically stable and must comply with post treatment monitoring. In our case multi dose methotrexate regimen i.e. $1.0 \mathrm{mg}$ $/ \mathrm{kg}$ body weight on days $1,3,5$ and 7 was adopted. Post treatment decline in weekly serum B HCG level showed the successful therapeutic intervention. The other treatments require an interventional radiologist, who might not always be available at hospital. If the patient comes to emergency department and the physician suspects a cervical pregnancy, the physician should consider whether the hospital is equipped to do these interventions, or whether a transfer might be necessary. In our case, patient was referred by physician at primary care centre on ultrasound suspicion of cervical ectopic pregnancy, which led to early interventional management and thus preserved patients' fertility without significant complication. In conclusion, it is important to carefully assess both the patients and the services available for the patient by making 
treatment decisions.

The effect of cervical ectopic pregnancies in the reproductive future has been evaluated in few studies. However, predisposing factors to cervical ectopic pregnancy are themselves associated with obstetric complications. Kung concluded that methotrexate, either alone or with adjuvant methods (with preservation of uterus), has not shown detrimental effects on subsequent reproductive and obstetric outcomes. Being the rare problem, all cases should be reported. ${ }^{16}$

\section{References}

1. Kochi, K.. Hidaka, T., Yasoshima, K. et al. Cervical pregnancy: a report of four cases. J Obstet Gynecol Res. 2014; 40: 603-606.

2. Kirk E, Condous G, Haider Z, Syed A, Ojha H, Bourne $\mathrm{T}$, The conservative management of cervical ectopic pregnancies. Ultrasound Obstet Gynecol. 2006; 27: 430-437.

3. Starita A, Di Miscia A, Evangelista S, Donadio F, Starita A. Cervical ectopic pregnancy: clinical review. Clin Exp Obstet Gynecol. 2006; 33: 47-9.

4. Leeman LM, Wendland CL. Cervical ectopic pregnancy. Diagnosis with endovaginal ultrasound examination and successful treatment with methotrexate. Arch Fam Med. 2000; 9: 72-7. (PubMed)

5. Cepni, Ocal P, Erkan S, Erzik B. Conservative treatment of cervical ectopic pregnancy with transvaginal ultrasound - guided aspiration and single dose methotrexate. Fertil Steril. 2004; 81: 1130-2. (PubMed)

6. Taylor, J.E. and Yalcinkaya, T.M. Munire Erman Akar. Successful conservative management of cervical ectopic pregnancy: a case series. Arch Gynecol Obstet. 2011; 283: 1215-1217.

7. Zakaria MA, Abdallah ME, Shavell VI, Berman JM, Diamond MP, Kmak DC. Conservative management of cervical ectopic pregnancy; utility of uterine artery embolization. Fertil Steril. 2011; 95: 872-6.

8. Raskin MM. Diagnosis of cervical pregnancy by ultrasound: a case report. Am Obstet J Gynecol. 1978; 130 (2): 234-5.

9. Sharma A, Ojha R, Mondal S, Chattopadhyay S, Sengupta $\mathrm{P}$. cervical intrmural pregnancy: report of a rare case. Niger Med J. 2013; 54: 271-3.
10. Spitzer D, Steiner H, Graf A, Zajc M, Staudach A. Conservative treatment of cervical pregnancy by curettage and local prostaglandin injection. Hum Repord. 1997; 12: 860-6. (PubMed)

11. Fylstra DL, Coffy MD. Treatment of cervical ectopic pregnancy with circlage, curettage and balloon tampnode. A report of three cases. J Repord Med. 2001;46: 71-4.( PubMed)

12. Modayil V, Ash A, Raio C. Cervical ectopic pregnancy diagnosed by point - of - care emergency department ultrasound. J Emerg Med. 2011; 41 (6): 655-7.

13. Ruano R, Reya F, Picano O,Chopin N, Pereiro PP, Benachi A, et al. Three - dimensional ultrasonographic diagnosis of a cervical pregnancy. Clinics (Sao Paulo) 2006; 61: 355-8. (PubMed)

14. Okamoto Y, Tanaka YO, Nishida M, Tsunoda M, Tsunoda H, Yoshikawa H, Itai Y. MR imaging of the uterine cervix; Imaging - pathologic correlation. Radiographics, 2003; 23: 425-45. (PubMed)

15. Practice Committee of American Society of Reproductive Medicine. Medical treatment of ectopic pregnancy: a committee opinion. Fertil Steril. 2013; 100: 638-44.

16. Jurkovic, D., Hacket, E., and Campbell, S. Diagnosis and treatment of early cervical pregnancy: a review and a report of two cases treated conservatively. Ultrasound Obstet Gynecol. 1996; 8: 373-380.

17. Chrestiana, D., Cheng, A.B., Panebianco, N.L. et al. Pitfalls in cervical ectopic pregnancy diagnosis by emergency medicine physicians using bedside ultrasonography: a case report. Am J Emerg Med. 2013;

18. Surampudi, K. A case of cervical ectopic pregnancy: successful therapy with methotrexate. J Obstet Gynecol India, 2012; 62: 1-3.

19. Polak G, Stachowicz N, Morawska D, Kotarski J. Treatment of cervical pregnancy with systemic methotrexate and KCI solution injection into the gestational sac Case report and review of literature. Ginekol Pol. 2011; 82: 386-9.

20. Kung FT, Chang SY. Efficacy of methotrexate treatment in viable and nonviable cervical pregnancies. Am J Obstet Gynaecol. 1999; 181: 1438-44. [PubMed]

21. Kung FT, Chang JC, Tsai YC Subsequent reproduction and obstetric outcome after methotrexate treatment of cervical pregnancy: a review of original literature and international collaborative follow up. Hum reprod. 19997; 12: 591-5. 